\title{
Wellens' sign: A reliable ECG sign for proximal left anterior descending coronary artery disease
}

\author{
Ragesh Panikkath MD, Deepa Panikkath MD, Anita Sultan MD, Leigh Ann Jenkins MD
}
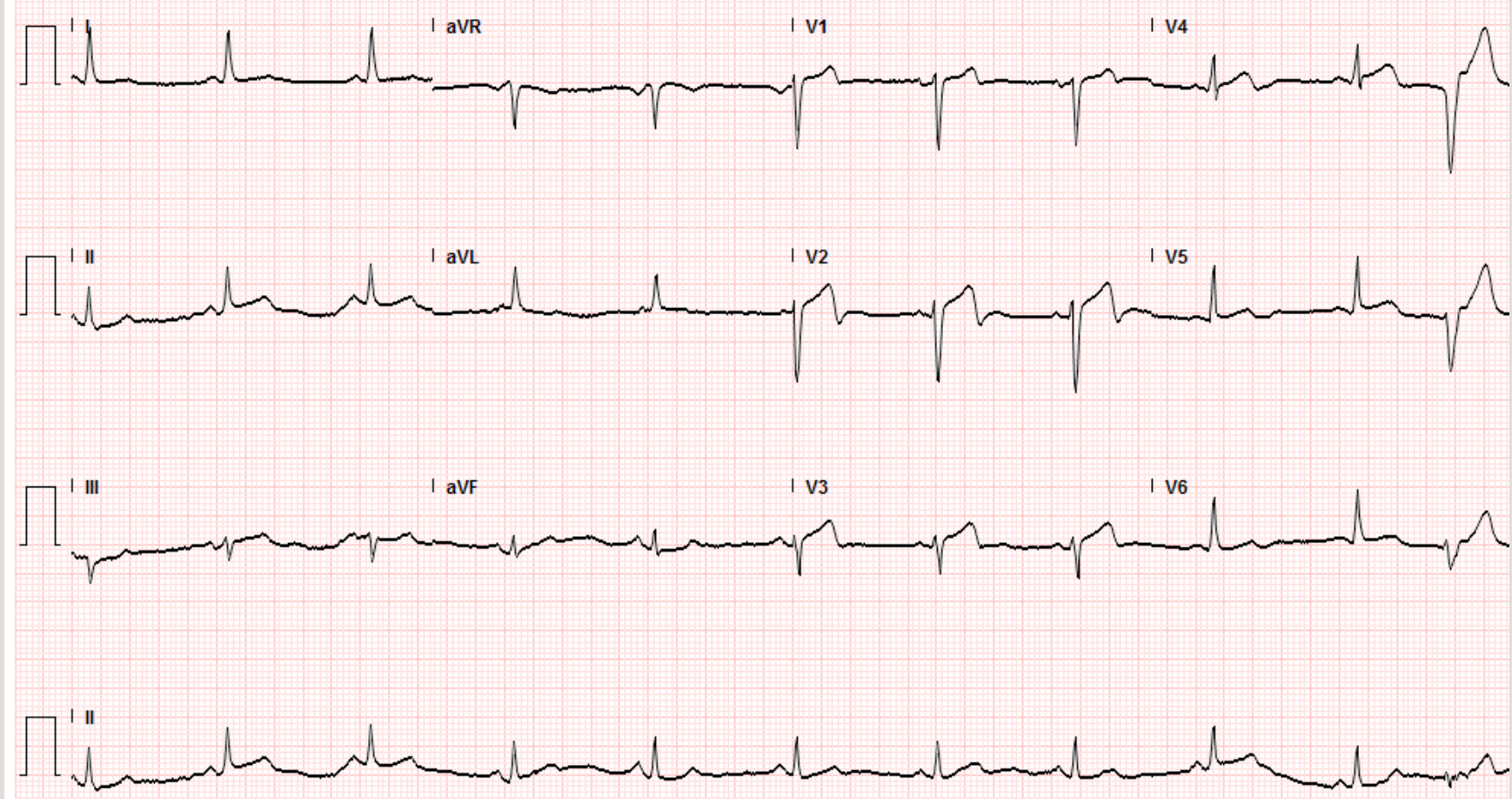

Figure ECG showing biphasic T wave inversions in V1, V2, and V3 consistent with Wellens' sign

\section{INTRODUCTION}

Wellens' sign is a reliable sign of significant proximal left anterior descending artery (LAD) disease, but awareness of this sign among internists, emergency room physicians, and family physicians is low. This ECG sign can be very helpful in identifying

Corresponding author: Ragesh Panikkath MD

Contact Information: Ragesh.Panikkath@ttuhsc.edu

DOI: $10.12746 /$ swrecc 2015.0309.116 patients who require cardiac catheterization among those who present with atypical chest pain.

\section{CAse Report}

A 54-year-old man with no past medical history presented with short episodes of sharp stabbing chest pain. This pain resolved completely with conservative management. However, his ECG showed biphasic $\mathrm{T}$ inversions in leads V1 and V2 (Wellens' 
sign). He underwent semi-urgent coronary angiography, which showed atherosclerosis with $99 \%$ stenosis in the proximal LAD. The other coronary arteries were free of any significant disease. A percutaneous intervention using a drug eluting stent opened the stenotic lesion. The patient did not have any recurrence of chest pain and was discharged the next day after the procedure. He remained asymptomatic on follow up. Here the identification of Wellens' sign helped identify the presence and location of the coronary artery disease and possibly prevented an anterior wall myocardial infarction.

\section{Conclusion}

Wellens' sign, otherwise known as the LAD T wave syndrome, was first described by Wellens and colleagues in 1982. ${ }^{1}$ These patients developed $T$ wave inversions in the symptom free period after angina. Although Wellens' sign has been reported in $14 \%-18 \%$ of patients with unstable angina, awareness of this sign among internists and primary care providers is low. It has a high specificity (89\%) and positive predictive value ( $86 \%)$ in detecting significant $(>70 \%)$ disease of the proximal LAD. This sign indicates a pre-infarction state of coronary artery disease and progression to anterior wall myocardial infarction occurs within a mean duration of 8.5 days after the onset of this sign. Seventy-five percent of patients who did not undergo revascularization developed anterior wall myocardial infarction within a few weeks. The second study from Wellens published in 1989 reported that all patients with this sign and angina had proximal LAD disease. ${ }^{2}$ This sign may not be present at the time of presentation; it may appear later after resolution of symptoms and may be missed if a follow up ECG is not obtained. Medical management is considered inadequate to prevent its progression. Some patients with Wellens' sign have developed ventricular tachycardia, myocardial infarction, and death during exercise stress tests. ${ }^{3}$ The proximal LAD supplies $45 \%-55 \%$ of ventricular myocardium. Massive anterior wall myocardial infarction which develops after occlusion of proximal LAD places the patients at risk of left ventricular dysfunction, heart failure, ventricular arrhythmias, and death. Awareness of Wellens' sign should help in the early detection of proximal LAD lesions and prevent such catastrophic events.

\section{KEY POINTS}

1. Wellens' sign is highly specific for the presence of proximal LAD disease, especially in patients with angina.

2. This sign often appears when patients are asymptomatic. Cardiac enzymes may be only minimally elevated.

3. This syndrome, if unrecognized, usually evolves into anterior wall myocardial infarction.

4. Although there are no recommendations available, stress tests should be avoided in these patients, as they have been reported to cause sudden death.

5. Physician awareness of this sign is essential for prompt identification and intervention.

\begin{abstract}
Author affiliation : All authors work in the Department of Internal Medicine at Texas Tech University Health Sciences Center in Lubbock, TX. Deepa Panikkath and Anita Sultan are internal medicine residents, Ragesh Panikkath is a fellow in cardiology, and Leigh Ann Jenkins is a faculty physician in cardiology.

Submitted: $10 / 21 / 2014$

Accepted: $11 / 9 / 2014$

Reviewers: Alejandro Perez-Verdia MD

Conflict of Interest: None

Published electronically: 1/15/2015
\end{abstract}

\section{References}

1. De Zwaan C, Bar FW, Wellens HJ. Characteristic electrocardiographic pattern indicating a critical stenosis high in the left anterior descending coronary artery in patients admitted because of impending myocardial infarction. Am Heart J 1982 Apr;103(4 Pt 2):730-6.

2. De Zwann C, Bar FW, Janssen HJ, et al. Angiographic and clinical characteristics of patients with unstable angina showing an ECG pattern indicating critical narrowing of the proximal LAD coronary artery. Am Heart J 1989; 117: 657-665.

3. Patel K, Alattar F, Koneru J, Shamoon F. ST-Elevation myocardial infarction after pharmacologic persantine stress test in a patient with Wellens' syndrome. Case Reports Emer Med 2014; 2014:530451. doi: 10.1155/2014/530451. Epub 2014 Apr 2. 